7-3-2009

\title{
Crack Identification by Multifractal Analysis of a Dynamic Rotor Response
}

Grzegorz Litak

University of Lublin, g.litak@pollub.pl

Jerzy T. Sawicki

Cleveland State University, j.sawicki@csuohio.edu

Follow this and additional works at: https://engagedscholarship.csuohio.edu/enme_facpub

Part of the Applied Mechanics Commons

How does access to this work benefit you? Let us know!

Publisher's Statement

This is the accepted version of the following article: Litak, G. and Sawicki, J.T. (2009). Crack identification by multifractal analysis of a dynamic rotor response. ZAMM - Journal of Applied Mathematics and Mechanics, 89(7), 587-592. doi: 10.1002/zamm.200900226., which has been published in final form at http://onlinelibrary.wiley.com/doi/10.1002/zamm.200900226/abstract

\section{Original Citation}

Litak, G. and Sawicki, J.T. (2009). Crack identification by multifractal analysis of a dynamic rotor response. ZAMM - Journal of Applied Mathematics and Mechanics, 89(7), 587-592. doi: 10.1002/ zamm.200900226.

This Article is brought to you for free and open access by the Mechanical Engineering Department at EngagedScholarship@CSU. It has been accepted for inclusion in Mechanical Engineering Faculty Publications by an authorized administrator of EngagedScholarship@CSU. For more information, please contact library.es@csuohio.edu. 


\title{
Crack identification by multifractal analysis of a dynamic rotor response
}

\author{
Grzegorz Litak $^{1, *}$ and Jerzy T. Sawicki ${ }^{2, * *}$ \\ ${ }^{1}$ Faculty of Mechanical Engineering, Lublin University of Technology, Nadbystrzycka 36, 20-618 Lublin, Poland \\ ${ }^{2}$ Cleveland State University, Department of Mechanical Engineering, Cleveland, OH 44115-2214, USA
}

\section{Introduction and the experimental procedure}

In many technical devices and machines possessing a rotor, their actual dynamic condition determines their safe and proper operation. Most rotating machines operate over extended periods of time in various temperature ranges, and they are often subjected to large loads. As a consequence of working conditions, their components are exposed to potential structural damage such as the shaft surface crack. Thus, proper health monitoring of rotating machines is an important task to extend the service life of particular components [1-6]. The experimental approach to this problem is based on examination of dynamical rotor responses and identification of differences between damaged and healthy rotors. The standard method uses the Fourier amplitudes of specific frequencies [7-11] or the wavelet approach [12-14]. For the rotor with a notch, which plays the role of a transverse crack, the strong local nonlinearity appears. Through the so called crack's breathing effect the parametric excitation with rotational frequency becomes the most important factor which influences the dynamic response. Magnetic actuators provide the additional excitation creating the new conditions of various kind of internal, and combinational resonances to distinguish cracked and healthy systems more easily $[7,8,15-18]$.

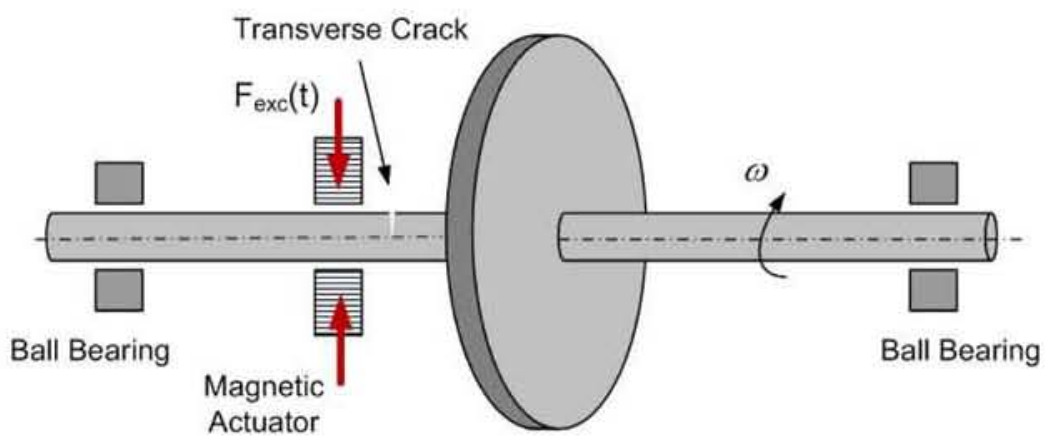

Fig. 1 (online colour at: www.zamm-journal.org) Schematic plot of the experimental cracked rotor. In the experiment the shaft diameter was $15.875 \mathrm{~mm}$ and the shaft length was $0.659 \mathrm{~m}$. The diameter of the active magnetic bearing rotors and radial actuator was $47.625 \mathrm{~mm}$. The disk has a diameter of $127 \mathrm{~mm}$ and a thickness of $12.7 \mathrm{~mm}$. The crack and magnetic actuator are depicted on the same side of the disk.

In the present paper we study the experimentally determined response of the test rotor system focusing on the effect of crack in a shaft. The test rotor consists of the shaft supported on two ball bearings and a single disk located midspan on a flexible shaft (Fig. 1). An active magnetic actuator placed near the disk produced an external harmonic force $F_{\text {ext }}(t)$ (see Fig. 1). The crack had the width of $0.94 \mathrm{~mm}$ and the depth of $40 \%$ of the shaft diameter. This system was investigated

* Corresponding author E-mail: g.litak@pollub.pl, Phone: +4881 538 1573, Fax: +4881 5250808

** E-mail: j.sawicki@csuohio.edu 
earlier by Sawicki et al. [7] where the main issue was the identification of Fourier amplitudes related to rotational frequency and its multiple values as $1 \mathrm{X}, 2 \mathrm{X}, 3 \mathrm{X}, 4 \mathrm{X}$ with and without magnetic excitation. The preliminary experimental results were predicted by the modelling with FEM.

The experimental time series of measured displacement, in the direction of $45 \mathrm{deg}$ from vertical one, for cracked and healthy rotors are presented in Fig. 2.

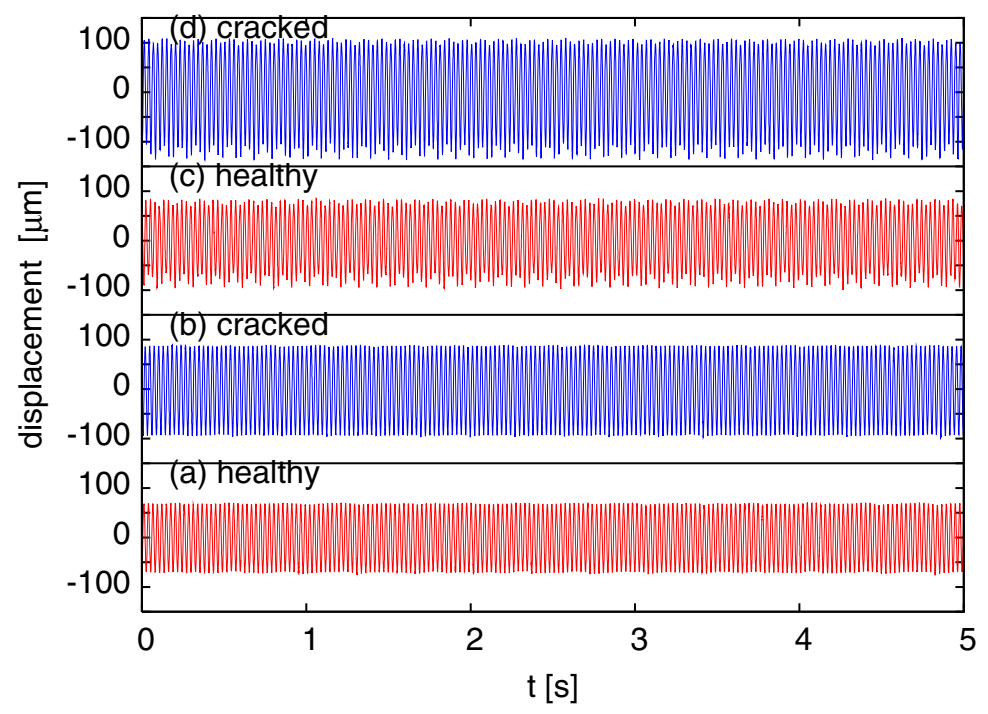

Fig. 2 (online colour at: www.zamm-journal.org) Experimental time series of the damaged and healthy machine for a spin velocity $\omega=2200 \mathrm{rpm}(36.7 \mathrm{~Hz})$ : (a,b) without external excitation; (c,d) with magnetic actuator frequency $3780 \mathrm{rpm}(63 \mathrm{~Hz})$.

In Figs. 2a,b we plotted the results of rotor vibrations due to residual unbalance for healthy (Fig. 2a) and cracked (Fig. 2b) rotors, respectively. One can see some increase of the amplitude of cracked rotor. More sophisticated changes can be seen in Figs. 2c-d where healthy (Fig. 2c) and cracked (Fig. 2d) rotors are subjected to the magnetic actuator applied harmonic force having amplitude of $200 \mathrm{~N} \mathrm{p-p} \mathrm{(peak-to-peak)} \mathrm{and} \mathrm{fixed} \mathrm{frequency} \mathrm{[7].}$

\section{Multifractal analysis}

In further studies we propose to use a multifractal analysis $[19,20]$ which appears to be a powerful tool to analyze the complexity of the nonlinear systems. This technique has been widely used in biological systems [21] but recently has been applied in engineering systems, e.g., to examine seismic sequences [22], and in combustion fluctuations in spark ignition engines $[23,24]$.

Following the multifractal procedure [20] we perform the Taylor expansion of the time series in the small vicinity time instant $t_{i}$

$$
p_{n}\left(t_{i}, \delta t\right)=a_{0}+a_{1} \delta t+\ldots+a_{n}(\delta t)^{n},
$$

where $p_{n}\left(t_{i}, \delta t\right)$ denotes a best polynomial approximation of degree $n, a_{0}, \ldots, a_{n}$ are local polynomial coefficients, $\delta t$ is a small time difference of the range of few sampling time steps.

The error between the examined function $p_{n}\left(t_{i}, \delta t\right)$ and the original time series $F\left(t_{i}^{\prime}\right)$, where $t_{i}^{\prime}=t_{i}+\delta t$, determines the local singularity in the time series. Namely,

$$
\left|F\left(t_{i}+\delta t\right)-p_{n}\left(t_{i}, \delta t\right)\right| \leq a_{h}(\delta t)^{h_{i}},
$$

where $a_{h}$ and $h_{i}$ are the coefficient and exponent related to the estimated power law (Eq. 2), respectively. Note that the degree of polynomial $n$ is an integer number less than the exponent $h_{i}$. $\delta t$ is a small time difference, while $a_{h}$ and $h_{i}$ are coefficient and exponent related to the estimated power law limiting function. The multifractal analysis of rotor vibrations is based on constructing a singularity spectrum $f(h)$ of all $h_{i}$ exponents providing a precise quantitative description of the system behaviour [20]. Formally, $h$ defines the Hölder exponent while the probability of its distribution $f(h)$ coincides with the Hausdorff dimension of a dynamical system. The results of our calculations are presented in Figs. $3 \mathrm{a}$ and $\mathrm{b}$ for the rotor response without external excitation and in the presence of magnetic actuators, respectively. The width of the spectrum $f(h), \Delta h=h_{\max }-h_{\min }$ is defined as the complexity measure of the system response while the $h_{0}$ which corresponds to the maximum of $f(h)$ indicates the correlation strength of vibrations. 

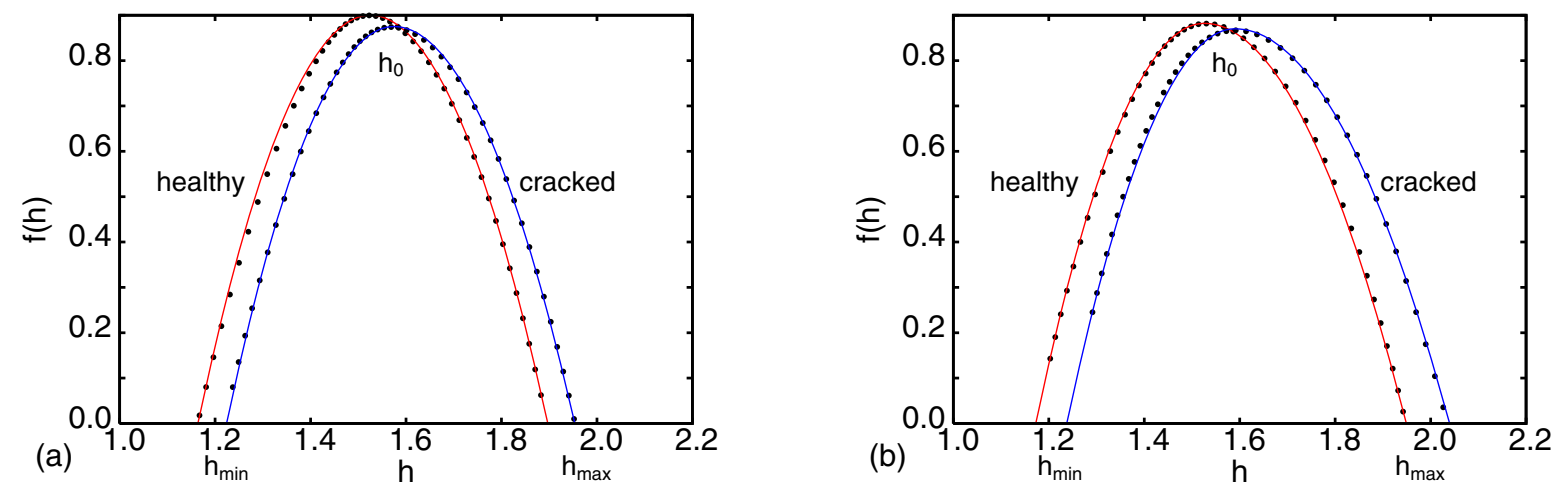

Fig. 3 (online colour at: www.zamm-journal.org) Spectra of the singularity exponents $f(h)$ for the system without external excitation (a) and in the presence of magnetic actuators (b) (see the corresponding time series in Fig. 2). The width of corresponding spectra $\Delta h$ and a peak position $h_{0}$ for healthy and cracked rotors: $\Delta h=0.729 \& 0.732, h_{0}=1.525 \& 1.575$ (a); $\Delta h=0.775 \& 0.803, h_{0}=1.525$ $\& 1.590$ (b).

Note that $h_{0}=0.5$ correspond to the uncorrelated Wiener process (Brownian motion). The wider the range of possible fractal exponents, the "richer" the process, in a structure. Larger $h_{0}$ in respect to $h_{0}=0.5$, means more correlated (or more regular) vibrations. In general, the higher the Hölder exponent, the smoother the time series.

In both plots we observe the shift of the spectrum to the right side (see the changes of $h_{0}$ position). This is related to more periodic behaviour of the cracked system. This effect is a manifestation of some extra coupling between vibration modes created by a defect in the rotor systems. Note also that in the rotor system without external excitation (Fig. 3a) the changes in the multifractal results in terms of $\Delta h$ are negligible (increase by $0.4 \%$ ) for the healthy and cracked rotors. Interestingly this difference of $\Delta h$, in case of the excited rotor, is noticeable (about one order higher increase - 3.6\%). The small difference in $\Delta h$ between healthy and cracked rotors is related to small increase of the response complexity. This small difference better visible for the system with magnetic excitation (Fig. 3b). On the other hand $h_{0}$ changes considerably in both cases (without and with magnetic excitation) shifting the exponent parabola $f(h)$ to the right hand side. These shifts are signaling the increase of correlation in the system response.

The above findings let us to conclude that by monitoring the changes in the multifractal spectra of a rotor we could identify crack (and other faults) in a rotor at an early stage in their development. The presented results show that the use of an multifractal analysis can identify the complex response of the system. The role of the magnetic actuator is crucial to increase visibility of the crack-induced effect.

\section{Relation to power series and wavelets}

In each of the cases considered above, an estimate of the most dominant fractal exponent, $h_{0}$ can be also obtained from the power spectrum of the time series. Let $P(\theta)$ denote the power spectrum function, with the frequency $\theta$. For an infinitely long mono-fractal time series, the decay of the power spectrum is described as

$$
P(\theta)=\theta^{-\gamma} \text {. }
$$

The exponent $\gamma$ can be obtained from the slope of the $\log P-\log \theta$ plot. In fact the value of $h_{0}$ can be also found from the following relation $[20,25]$ :

$$
h_{0}=(\gamma-1) / 2 \text {. }
$$

The corresponding power spectra are presented in Fig. 4. Note, the difference in the inclination slopes are related to variation of $h_{0}$ in all examined cases. Evidently for cracked rotor we observe larger slope $\beta$ corresponding to larger $h_{0}$. This is connected to the more pronounce appearance of nonlinear resonances in the cracked system. The strong nonlinearity introduced by a crack appearance leads to more effective effect of frequency vibration frequency entrainment in respect to the rotational velocity of the shaft. Consequently the whole spectrum is strictly changed reflecting the difference in slope.

The Hölder exponent is also present in the wavelet structure. Wavelet transforms make use of scaling functions that have the property of being localized in both time and frequency. A scaling coefficient $s$ characterizes and measures the width of a wavelet. Given a signal $F(t)$, the continuous wavelet transform (CWT) of $F(t)$ is defined by a convolution

$$
W_{s, t_{0}}(F)=\int_{-\infty}^{\infty} \frac{1}{s} \psi\left(\frac{t-t_{0}}{s}\right) F(t) d t,
$$



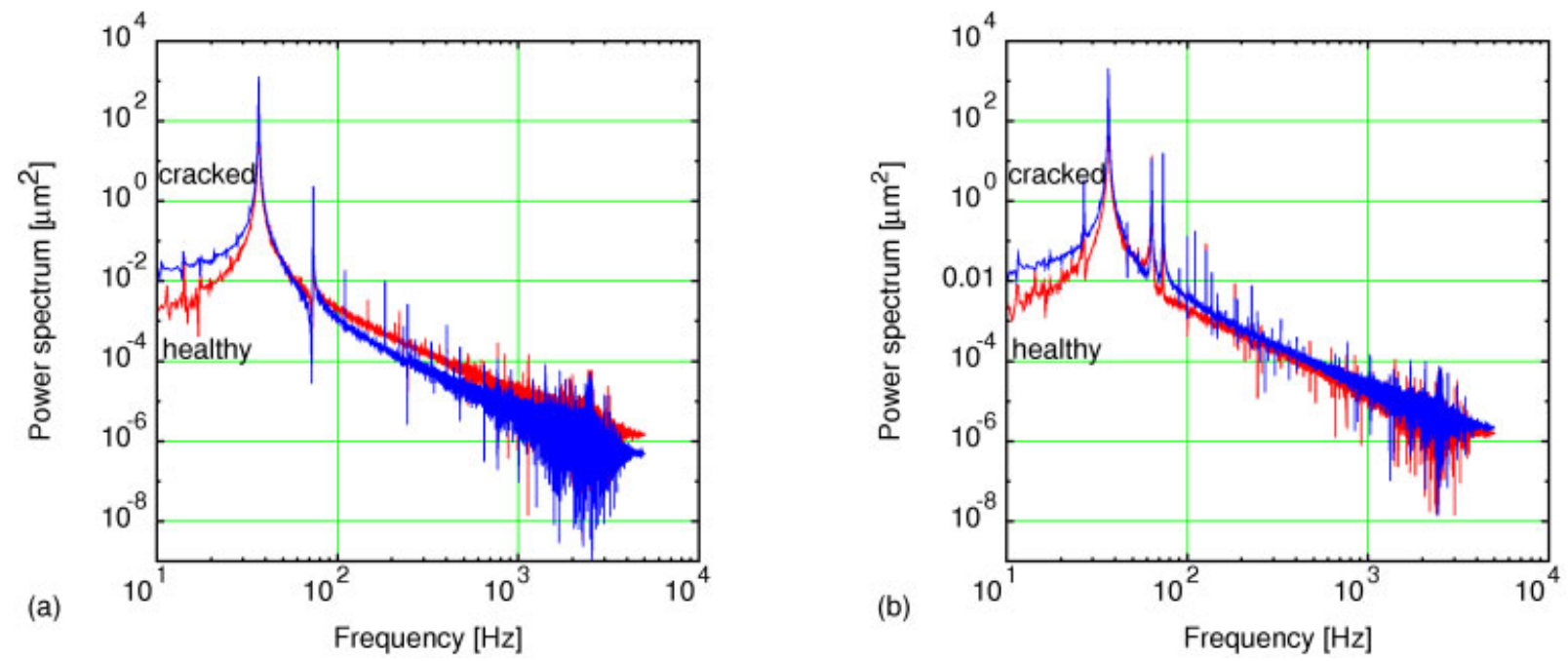

Fig. 4 (online colour at: www.zamm-journal.org) Power spectra of the logarithmic scale without external excitation (a) and in the presence of magnetic actuators (b)

For $F\left(t_{i}\right)$ satisfying the relation (Eq. 1) the wavelet density is given by [25]

$$
W_{s, t_{0}}(F) \sim|s|^{h\left(t_{0}\right)} \text {. }
$$

For clarity, in Fig. 5 we present the corresponding wavelet power spectra $P_{W}(s, t)$

$$
P_{W}(s, t)=\left|W_{s, t}\right|^{2}
$$

calculated for all examined cases (as in Fig. 2). In calculations we used a complex Morlet wavelet as the mother wavelet. A Morlet wavelet consists of a plane wave modulated by a Gaussian function and is described by

$$
\psi(\eta)=\pi^{-1 / 4} \mathrm{e}^{\mathrm{i} \theta_{0} \eta} \mathrm{e}^{\eta^{2} / 2},
$$

where $\theta_{0}$ (in our case $\theta_{0}=6$ ) is the center frequency, also referred to as the order of the wavelet [26]. In each of plots (Figs. 5a-d) the maximum power spectrum is related the period 270 strictly related to frequency of rotational motion of the rotor $(36.7 \mathrm{~Hz})$. In Fig. 5a,b one can easily see very fast fluctuation of $P_{W}$ for the period about $0.0128 \mathrm{~s}$. Additional modulation is caused by the magnetic excitation. Note that such a modulation was visible in Figs. 2c,d. In Figs. 5c and d it can be observed as the periodic corrugation of the constant $P_{W}$ cross-sections (Figs. 5c,d) in the period region around $0.0128 \mathrm{~s}$. Note that this kind of modulation reflects local critical exponent $\left(h_{i}\right)$ fluctuations of $W_{s, t_{0}}$. Comparing pairs of cracked and healthy cases (Fig. 5a,b or Fig. 5c,d) one can conclude that these fluctuations are noticeable stronger for cracked rotors (Figs. $5 \mathrm{~b}$ and d, respectively). Furthermore, the difference is more transparent for the system with the active magnetic actuator (see Figs. $5 \mathrm{c}$ and d).

\section{Summary and conclusions}

In summary, we conclude that the presented approach has some advantages, enabling quantification of the effect of crack using the measure of correlation $h_{0}$ and complexity $\Delta h$. The alternative way is to analyze the corresponding Fourier spectrum [7,8], however in this approach one needs to examine longer time series. Note that the notch of $1 \mathrm{~mm}$ does not posses all features of a real crack. For its width of $1 \mathrm{~mm}$ the breathing effect becomes almost marginal.

It should be noted that the multifractal properties are strictly related to the scaling properties of power and wavelet spectra related to stronger frequency representation around resonances in case of a cracked rotor and the characteristic decay of amplitudes in higher frequencies. It has been shown that the multifractal analysis can be a useful approach in rotor diagnostics. The main effect is the shift of the average Hölder exponnent $h_{0}$ (Fig. 3a,b) related to the presence of crack in a rotor. Additionally, in the presence of magnetic excitation, the measure of complexity $\Delta h$ can be applied.

However to produce a robust multifractal condition monitoring technique more tests are necessary. Especially one could perform various excitation type by using magnetic actuators and examine different locations or shapes of cracks. We are going to continue the present studies by using multifractals in the rotor system of different notch depths and widths. The result of such investigations will be reported in the next paper. 

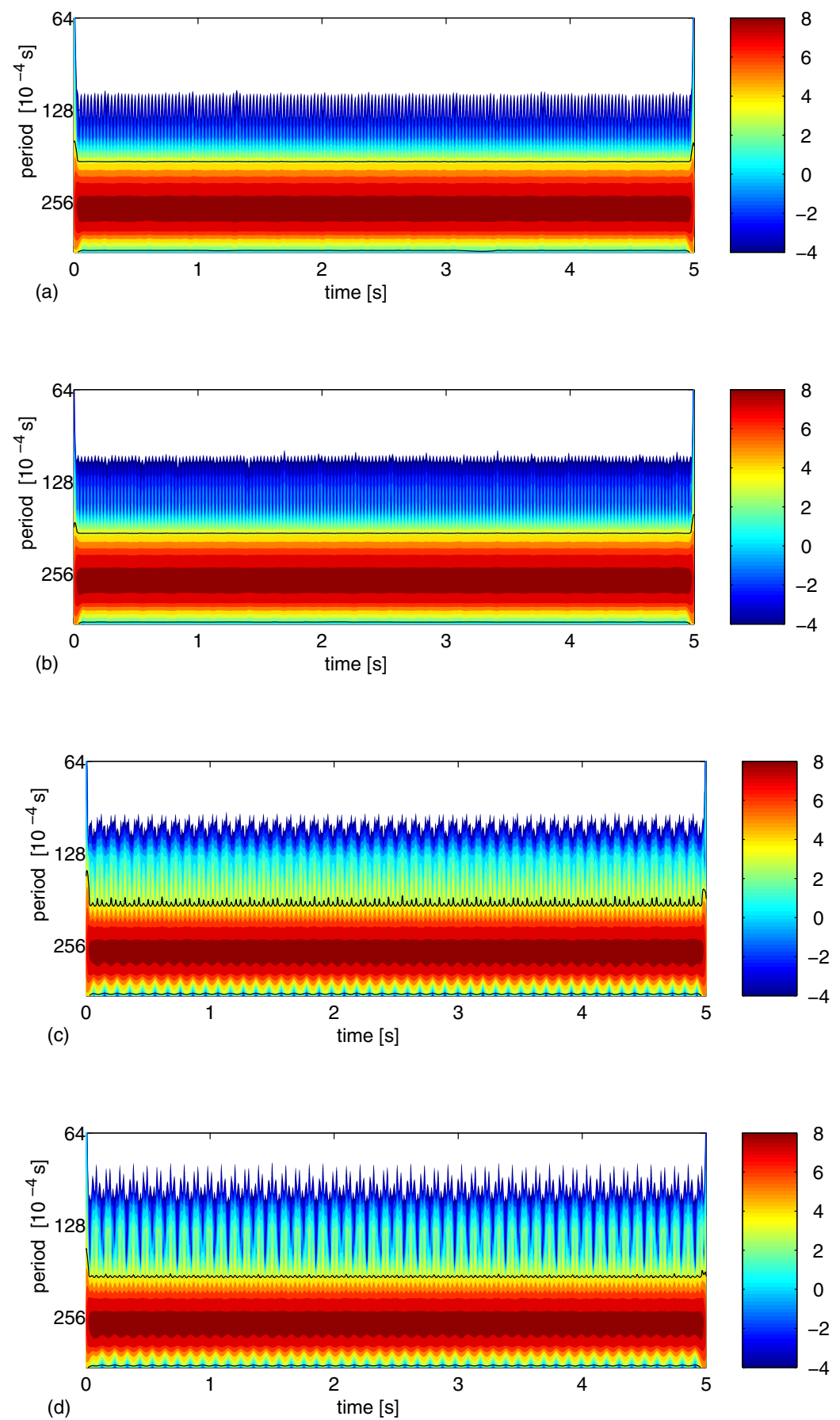

Fig. 5 (online colour at: www.zamm-journal.org) Wavelet spectra of a rotor without external excitation $(a, b)$ and in the presence of magnetic actuators (c,d). The crack is present in b and d plots, respectively. In CWT calculations a Morlet wavelet was used. The colours are related to the magnitudes of $P_{W}(s, t)$ (Eq. 6) according to the $\operatorname{logarithmic} \operatorname{scale} \log _{2}$ (see right panels marked with the corresponding scale exponents).

Acknowledgements This research has been supported by NASA's Research Opportunities in Aeronautics, grant number NNX08AC31A. and by the Polish Ministry for Science and Higher Education. The authors would like to thank the graduate students Alex Pesch and Adam Wroblewski at Cleveland State University for their help in obtaining experimental data. 


\section{References}

[1] R. A. Gasch, Survey of the dynamic behavior of a simple rotating shaft with a transverse crack, J. Sound Vib. 160, 313-332 (1993)

[2] A. D. Dimarogonas, Vibration of cracked structures: a state-of-the-art review, Eng. Fract. Mech. 55, 831-857 (1996).

[3] A. W. Doebling, C. R. Farrar, M. B. Prime, and D. W. Shevitz, Damage identification and health monitoring of structural and mechanical systems from changes in their vibration characteristics: a literature review, in Report LA-13070-MS, Los Alamos National Laboratory, Los Alamos, New Mexico, 1996.

[4] G. Sabnavis, R. G. Kirk, M. Kasarda, and D. Quinn, Cracked shaft detection and diagnostics: a literature review, Shock Vibr. Dig. 36, 287-296 (2004).

[5] Y. Ishida, Cracked rotors: industrial machine case histories and nonlinear effects shown by simple Jeffcott rotor, Mech. Syst. Signal Proc. 22, 805-817 (2008).

[6] F. F. Ehrich, Handbook of Rotordynamics, revised edition, (Kriegar Publishing Company, Malabar, Florida, 1999).

[7] J. T. Sawicki, M. I. Friswell, A.H. Pesh, and A. Wroblewski, Condition monitoring of rotor using active magnetic actuator, in Proceedings of ASME Turbo Expo 2008: Power for Land, Sea and Air, GT2008, June 9-13, 2008, Berlin, Germany.

[8] G. Mani, D. D. Quinn, and M. Kasarda, Active health monitoring in a rotating cracked shaft using active magnetic bearings as force actuators, J. Sound Vib. 294, 454-465 (2006).

[9] C. T. Zheng, Vibration of a rotor system with a switching crack and detection of crack, J. Eng. Gas Turb. 20, 149-154 (1998).

[10] M.-C. Yu, and S.-S. Huang, In-plane vibration and crack detection of a rotating shaft-disk containing a transverse crack, J. Vib. Acoust. 120 551-555 (1998).

[11] S.A. Adewusi and B.O. Al-Bedoor, Analysis of vibration signals of an overhang rotor with a propagating transverse crack, J. Sound Vib. 246, 777-793 (2001).

[12] S. Prabhakar, A. S. Sekhar, and A. R. Mohanty, Detection and monitoring of cracks in a rotor-bearing system using wavelet transform, Mech. Syst. Signal Proc. 15 447-450 (2001).

[13] A. S. Sekhar, Crack detection through wavelet transform for a run up rotor, J. Sound Vib. $259461-472$ (2003).

[14] J. T. Sawicki, A. K. Asok, and G. Litak, Multiresution wavelet analysis of cracked rotor, J. Rotat. Machin. (2009) in press.

[15] J. T. Sawicki, Rotor crack detection using active magnetic bearings, Solid State Phenom. 144, 9-15 (2009).

[16] J. T. Sawicki, X. Wu, G. Baaklini, and A. L. Gyekenyesi, Vibration-based crack diagnosis in rotating shafts during acceleration through resonance, in: Proceedings of SPIE 10th Annual International Symposium on Smart Structures and Materials, San Diego, California, 2003.

[17] X. Wu, J. T. Sawicki, M. I. Friswell, and G. Baaklini, Finite element analysis of coupled lateral and torsional vibration of a rotor with multiple cracks, in: Proceedings of ASME Turbo Expo Conference, Reno-Tahoe, Nevada, USA, Paper GT2005-68839, 2005.

[18] S. H. Imam, R. Azzaro, J. Bankert, and J. Scheibel, Development of on-line rotor crack detection and monitoring system, J. Vib. Acoust. Stress Reliab. Des. 111, 241-250 (1989).

[19] G. Brown, G. Michon, and J. Peyriére, On the multifractal analysis of measures, J. Stat. Phys. 66, 775-790 (1992).

[20] H. Riedi, 2001, Multifractal Processes, in: Long Range Dependence: Theory and Applications, edited by P. Doukhan, G. Oppenheim, and M. S. Taqqu, (Birkhäuser, Cambridge) p. 625.

[21] A. L. Goldberger, L. A. N. Amaral, L. Glass, J. M. Hausdorff, P. Ch. Ivanov, R. G. Mark, J. E. Mietus, G. B. Moody, C.-K. Peng, and H. E. Stanley, 2000: PhysioBank, physioToolkit, and physioNet: Components of a new research resource for complex physiologic signals, Circulation 101, E215-E220 (2000).

[22] L. Telesca and V. Lapenna, Measuring multifractality in seismic sequences, Tectonophysics 423, 115-123 (2006).

[23] A. K. Sen, G. Litak, T. Kaminski, and M. Wendeker, Multifractal and statistical analyses of heat release fluctuations in a spark ignition engine, Chaos 18, 033115 (2008).

[24] G. Litak, M. Geca, B.-F. Yao, and G.-X. Li, Indicated mean effective pressure oscillations in a natural gas combustion engine, Z. Naturforsch. 64a, 393-398 (2009).

[25] B. J. West, N. Scafetta, W. H. Cooke, and R. Balocchi, Influence of progressive central hypovolemia on multifractal dimension of cardiac interbeat intervals, Ann. Biomed. Eng. 23, 1077-1087 (2004).

[26] C. Torrence and G. P. Compo, A practical guide to wavelet analysis, Bull. Am. Meteor. Soc. 79, 61-78 (1998). 